\title{
ON THE RATIONALITY OF THE VARIETY \\ OF SMOOTH RATIONAL SPACE CURVES WITH FIXED DEGREE AND NORMAL BUNDLE
}

\author{
EDOARDO BALLICO ${ }^{1}$
}

\begin{abstract}
Let $\dot{S}_{n, a}$ be the variety of smooth, rational curves of degree $n$ in $\mathbf{P}_{3}$ whose normal bundle has a factor of degree $2 n-1+a$ and a factor of degree $2 n-1-a$. In this paper we prove that $\tilde{S}_{n, a}$ is rational if $n-a$ is even and $a>0$.
\end{abstract}

We work over $\mathbf{C}$. Let $\tilde{S}_{n, a} \subset$ Hilb $\mathbf{P}_{3}$ be the set of smooth, rational curves in $\mathbf{P}_{3}$ of degree $n$ whose normal bundle splits with a summand of degree $2 n-1-a$ and another of degree $2 n-1+a$. Eisenbud and Van de Ven [1,2] proved that for $0 \leqslant a \leqslant n-4, \tilde{S}_{n, a}$ is not empty, irreducible and of dimension $4 n-2 a+1$ (if $a>0)$. Let $S_{n, a}$ be the set of embeddings $f: \mathbf{P}_{1} \rightarrow \mathbf{P}_{3}$ with $f\left(\mathbf{P}_{1}\right) \in \tilde{S}_{n, a}$. They proved in [2] that $S_{n, a}$ is irreducible, rational and, if $a>0$, of dimension $4 n-2 a+4$. $\operatorname{PGL}(2)=\operatorname{Aut}\left(\mathbf{P}_{1}\right)$ acts naturally on $S_{n, a}$ without fixed points. $\tilde{S}_{n, a}$ is the quotient of $S_{n, a}$ by this action and the natural map $S_{n, a} \rightarrow \tilde{S}_{n, a}$ makes $S_{n, a}$ a principal locally isotrivial bundle over $\tilde{S}_{n, a}$ with structural group PGL(2) (see Serre [6] for this notion).

In the introduction to [2] Eisenbud and Van de Ven raised the question of the rationality of $\tilde{S}_{n, a}$. Here we prove the following

THEOREM. If $a>0$ and $n-a$ is even, then $\tilde{S}_{n, a}$ is rational.

The proof of this theorem uses only the construction in [2, §5], elementary properties of conic bundles (or $\mathbf{P}_{1}$-bundles) with smooth fibers and smooth base, and the definition of stably rational varieties due to Kollar and Schreyer [4]. An irreducible variety $V$ is said to be stably rational of level $k$ if $V \times \mathbf{P}_{k}$ is rational. For the elementary properties of conic bundles we need to see Serre [6]; we also found useful $[3,5]$.

We write $\tilde{S}_{n}$ for the variety of smooth, rational curves of degree $n$ in $\mathbf{P}_{3}$ and $S_{n}$ for the set of embeddings of degree $n$ of $\mathbf{P}_{1}$ into $\mathbf{P}_{3} . S_{n}$ is rational and $S_{n} \rightarrow \tilde{S}_{n}$ is a principal locally isotrivial bundle with structure group PGL(2). Since $S_{n}$ (resp. $S_{n, a}$ ) is rational, if the natural map $p: S_{n} \rightarrow \tilde{S}_{n}$ (resp. $S_{n, a} \rightarrow \tilde{S}_{n, a}$ ) has a rational section,

Received by the editors September 6, 1983 and, in revised form, October 13, 1983.

1980 Mathematics Subject Classification. Primary 14M20: Secondary 14D20, 14F05, 14H45.

Ke' nords and phrases. Rational curve, projective space, rational variety. normal bundle, degree, irreducible varicty, principal bundle, projective group.

' Supported in part by CNR (Italy) at Brandeis University. 
then $\tilde{S}_{n}$ (resp. $\tilde{S}_{n, a}$ ) is stably rational of level 3 . The rationality of $S_{n, a}$ was proved in [2, p. 97].

Lemma 1. Assume $n$ odd. Then for every $x \in \tilde{S}_{n}$, there exists a rational section of $p$ defined at $x$.

Proof. Since $\tilde{S}_{n}$ is contained in Hilb $\mathbf{P}_{3}$, we have a universal curve $C \rightarrow \tilde{S}_{n}$ with an inclusion $i: C \rightarrow \tilde{S}_{n} \times \mathbf{P}_{3}$ over $\tilde{S}_{n}$. C is a conic bundle with a smooth base. Since $n$ is odd, this conic bundle is locally trivial in the Zariski topology [2]. Thus there is a neighborhood $U$ of $x$ and an $U$-isomorphism $h: U \times \mathbf{P}_{1} \rightarrow C$. The map $i \circ h$ gives the section of $p$ defined on $U$.

We write $R_{n}$ for the set of maps of degree $n$ of $\mathbf{P}_{1}$ into $\mathbf{P}_{3}$. Again PGL(2) acts on $R_{n}$ and we write $\tilde{R}_{n}$ for its quotient. Since we are interested only at birational geometry, there is no problem here; we can substitute $R_{n}$ with $S_{n}$ if we want. In [2] a key point was the map $G: S_{n, a} \rightarrow R_{n-a-1}(a>0)$ constructed in the following way. Fix $f \in S_{n, a}$.

$$
N_{f}:=f^{*}\left(N_{f\left(\mathbf{P}_{1}\right) / \mathbf{P}_{3}}\right) \cong \mathcal{O}_{\mathbf{P}_{1}}(2 n-1-a) \oplus \mathcal{O}_{\mathbf{P}_{1}}(2 n-1+a)
$$

is a quotient of $f^{*}\left(T \mathbf{P}_{3}\right)$. Thus the subline bundle $\mathcal{O}_{\mathbf{P}_{1}}(2 n-1+a)$ defines a rank-2 subbundle $V_{f}$ of $f^{*}\left(T \mathbf{P}_{3}\right)$. The map $G(f): \mathbf{P}_{1} \rightarrow \mathbf{P}_{3}$ is constructed by taking for $G(f)(t)$ the plane in $\mathbf{P}_{3}$ which is determined by $V_{f, t} \subset T \mathbf{P}_{3, f(t)}$. Note that the map $G$ descends to a map $\tilde{G}: \tilde{S}_{n, a} \rightarrow \tilde{R}_{n-a-1}$ such that, for $0<a \leqslant n-4$ we have the following commutative diagram:

$$
\begin{array}{cccc}
S_{n, a} & \stackrel{G}{\rightarrow} & R_{n-a-1} & \\
\downarrow q & & \downarrow g & 0<a \leqslant n-4 . \\
\tilde{S}_{n, a} & \stackrel{\dot{G}}{\rightarrow} & \tilde{R}_{n-a-1} &
\end{array}
$$

Eisenbud and Van de Ven [2, p. 97] proved that $G$ is birationally the projection of a product with fiber rational of dimension $2 a+5$. If $n-a$ is even, by Lemma $1 g$ has a rational section. Thus $\tilde{R}_{n-a-1}$ is stably rational of level $3, \tilde{G}$ is birationally a product with fiber $\mathbf{P}_{2 a+5}$ and $\tilde{S}_{n, a}$ is rational. This concludes the proof of Theorem 1 .

If $n-a$ is odd, $a>0$, we do not know very much. A trick easily gives the following

Proposition 1. Assume $a>0$. Then $\tilde{S}_{n, a}$ is covered by rational subvarieties of codimension 2.

Proof. Fix a point $O \in \mathbf{P}_{1}$ and a point $P$ in $\mathbf{P}_{3}$. Let $A_{n}$ be the set of embeddings $f$ of $\mathbf{P}_{1}$ into $\mathbf{P}_{3}$ with $f(O)=P$ and $\operatorname{deg}\left(f\left(\mathbf{P}_{1}\right)\right)=n$. $A_{n}$ is rational. The affine group of projective transformations of $\mathbf{P}_{1}$ fixing $O$ acts on $A_{n}$ and let $\tilde{A}_{n} \subset$ Hilb $\mathbf{P}_{3}$ be the quotient. $A_{n}$ is the subset of $S_{n}$ formed by curves through $P$. The map $A_{n} \rightarrow \tilde{A}_{n}$ has always a rational section. This follows from the speciality of the affine group [3, Lemme 2.3]. Alternatively the restriction to $\tilde{A}_{n}$ of the conic bundle of Lemma 1 comes from a vector bundle since the point $P$ defines a line bundle on $p^{-1}\left(\tilde{A}_{n}\right)$ with degree one on every fiber. 
Thus $\tilde{A}_{n-a-1}$ is stably rational of level 2 and $\tilde{G} \mid \tilde{G}^{-1}\left(\tilde{A}_{n-a-1}\right)$ has a rational section. Thus $\tilde{G}^{-1}\left(\tilde{A}_{n-a-1}\right)$ is a rational subvariety of codimension 2 of $\tilde{S}_{n, a}$.

For $a=0$ the same method gives only that $\tilde{S}_{n}$ is covered by codimension 2 subvarieties which are stably rational of level 2 .

\section{REFERENCES}

1. D. Eisenbud and A. Van de Ven, On the normal bundles of rational space curves, Math. Ann. 256 (1981), 453-463.

2. $\ldots$ On the variety of smooth rational space curves with given degree and normal bundle, Invent. Math. 67 (1982), 89-100.

3. A. Hirschowitz and M. S. Narasimhan, Fibres de' $t$ Hooft et application, Enumerative Geometry and Classical Algebraic Geometry, Progress in Math., vol. 24, Birkhäuser, Basel, 1982.

4. J. Kollar and F. O. Schreyer, The moduli of curves is stably rational for $g \leqslant 6$ (preprint).

5. P. S. Newstead, Comparison theorems for conic bundles, Math. Proc. Cambridge Philos. Soc. 90 (1981), 21-31.

6. J. P. Serre, Espaces fibres algébriques, Séminaire C. Chevalley 1958 Anneaux de Chow et Applications, Exp. 1, Secretariat mathematique, Paris, 1958.

Department of Mathematics, Scuola Normale Superiore, 56100 Pisa, Italy 